\title{
Strong duality with super efficiency in set-valued optimization
}

\author{
Guolin Yu \\ Institute of Applied Mathematics, North Minzu University, Yinchuan, Ningxia 750021, P. R. China. \\ Communicated by Y. H. Yao
}

\begin{abstract}
This paper is devoted to the study of four dual problems of a primal vector optimization problem involving nearly subconvexlike set-valued mappings. For each dual problem, a strong duality theorem with super efficiency is established. The strong duality result can be expressed as follows: starting from a super minimizer of the primal problem, a super maximizer of the dual problem can be constructed such that the corresponding objective values of both problems are equal. The results improve the corresponding ones in the literature. (c)2017 All rights reserved.
\end{abstract}

Keywords: Super efficiency, Henig proper efficiency, nearly subconvexlike set-valued mappings, set-valued optimization, strong duality.

2010 MSC: 90C29, 90C46, $26 \mathrm{~B} 25$.

\section{Introduction}

It is well-known that in vector optimization, various notions of proper efficiency are introduced to eliminate anomalous efficient solutions. The reader is referred to $[7,8]$ for the comprehensive survey of concepts of proper efficiency. Among these notions of proper efficiency, super efficiency, which was introduced by Borwein and Zhuang [2] for convex vector optimization in normed spaces, has been widely investigated over the last couple of decades. This is because that super efficiency refines the notions of efficiency and other kinds of proper efficiency, and provides a concise (and equivalent) scalar characterization. Later, Zheng [23] extended the concept of super efficiency from normed spaces to locally convex topological vector spaces. Some achievements related to super efficiency in vector optimization were developed in terms of scalarization, Lagrange multiplier, Lagrange duality, saddle point, topological property, etc., see [3, 6, 9, 10, 12, 13, 19-21]. Recently, Zheng et al. [24] and Bao and Mordukhovich [1] dealt with super efficiency in Banach spaces by employing tools of variational analysis. However, there exist many ordered locally convex topological vector spaces which are not normable, even not metrizable (see [4]). This paper will study on super efficiency in the framework of more general spaces, locally convex topological vector spaces.

Since duality assertions allow to study a minimization problem through a maximization problem and to know what one can expect in the best case. At the same time, duality has resulted in many

Email address: guolin_yu@126.com (Guolin Yu)

doi:10.22436/jnsa.010.06.37 
applications within optimization, and it has provided many unifying conceptual insights into economics and management science. In vector optimization, it is often said that strong duality holds between primal and dual problems, if a weakly efficient solution of a primal problem is a weakly efficient solution of the dual problem and such that the corresponding objective values of the primal and dual problems are equal. If weakly efficient solutions are replaced by properly efficient solutions, then it is said that strong duality with proper efficiency holds between the primal and dual problems (see [5, 15, 16]). However, to the best of our knowledge, strong duality with super efficiency is not considered. In this paper, we will try to establish strong duality theorems for super efficiency.

In recent years, several notions of generalized convexity for set-valued mappings are introduced and are proved to be useful for optimization theory and related topics, for instance, see [14, 22]. Among them, the notion of nearly cone-subconvexlikeness seems to be more general and is successfully applied to super efficiency in set-valued optimization (see [19-21]). The notion of generalized convexity we use in the current paper is that of nearly cone-subconvexlikeness.

In this paper, we will consider strong duality with super efficiency for vector optimization involving nearly cone-subconvexlike set-valued mappings in the framework of locally convex topological spaces. The paper is arranged as follows. In Section 2, we collect some notions and results that will be used later. In Section 3, four dual models are introduced for a primal set-valued optimization problem, and weak and strong duality theorems with super efficiency are established, respectively.

\section{Notations and preliminaries}

Throughout the paper, if not otherwise specified, it is assumed that $X, Y$ and $Z$ are locally convex Hausdorff topological vector spaces with topological duals, denoted by $X^{*}, Y^{*}$ and $Z^{*}$, receptively. For any $x \in X$ and $x^{*} \in X^{*}$, the canonical form between $X$ and $X^{*}$ is denoted by $\left\langle x^{*}, x\right\rangle$. Given a set $A$ in $X$, the symbols $\operatorname{cl}(A)$ and $\operatorname{int}(A)$ stand for the closure and interior of $A$, respectively, and the cone generated by $A$ is denoted by

$$
\operatorname{cone}(A)=\left\{\lambda a: a \in A, \lambda \in \mathbb{R}_{+}\right\},
$$

where $\mathbb{R}_{+}$denotes the set of nonnegative real numbers. Let $\mathcal{C} \subset \mathrm{Y}$ and $\mathcal{K} \subset \mathrm{Z}$ be pointed closed convex cones and $\operatorname{int}(\mathcal{K}) \neq \emptyset$, where $\emptyset$ denotes the empty set. The cone $\mathcal{C}$ induces an order relation on $Y$ as follows: for $y_{1}, y_{2} \in Y$,

$$
\begin{array}{lll}
y_{1} \leqslant \mathcal{e} y_{2} \text { if and only if } & y_{2}-y_{1} \in \mathcal{C} \backslash\{0\}, \\
y_{1} \nless \mathcal{e} y_{2} \text { if and only if } \quad y_{2}-y_{1} \notin \mathcal{C} \backslash\{0\}
\end{array}
$$

Let $L(X, Y)$ be the family of linear continuous operator from $X$ into $Y$. By $L_{+}(\mathcal{K}, \mathcal{C})$, we denote the set of all continuous linear mappings $\mathrm{T}: Z \rightarrow Y$ such that $\mathrm{T}(\mathcal{K}) \subset \mathcal{C}$, i.e., $\mathrm{T}(\mathrm{k}) \in \mathcal{C}$ for all $k \in \mathcal{K}$. A convex subset $\Theta$ of $\mathcal{C}$ is a base of $\mathcal{C}$ if $0 \notin \operatorname{cl}(\Theta)$ and $\mathcal{C}=\operatorname{cone}(\Theta)$. We write

$$
\mathcal{C}^{+}=\left\{\mathrm{y}^{*} \in \mathrm{Y}^{*}:\left\langle\mathrm{y}^{*}, \mathrm{c}\right\rangle \geqslant 0, \forall \mathrm{c} \in \mathcal{C}\right\},
$$

and similarly for $\mathcal{K}^{+}$. Let $\Theta$ be a base of $\mathcal{C}$. Define

$$
\Theta^{s t}:=\left\{y^{*} \in Y^{*}: \text { there exists } t>0 \text { such that }\left\langle y^{*}, \theta\right\rangle \geqslant t, \forall \theta \in \Theta\right\} .
$$

Lemma 2.1 ([19]). Let $\Theta$ be a base of $\mathcal{C}$. Then $\Theta^{\text {st }}$ enjoys the following properties:

(i) Let $\mathrm{y}^{*} \in \mathrm{Y}^{*} \backslash\{0\}$. Then, $\mathrm{y}^{*} \in \Theta^{\text {st }}$ if and only if there exists a neighborhood $\mathrm{V}$ of 0 in $\mathrm{Y}$ such that $\left\langle\mathrm{y}^{*}, \mathrm{y}\right\rangle<0$, for any $\mathrm{y} \in \mathrm{V}-\Theta$.

(ii) If $\Theta$ is bounded, then $\Theta^{\text {st }}=\operatorname{int}\left(\mathrm{C}^{+}\right)$.

Next, let us introduce some notions of efficiency in vector optimization. Let $A$ be a nonempty subset 
of $Y$ and $\bar{y} \in A . \bar{y}$ is said to be a minimizer [maximizer] of $A$ with respect to $\mathcal{C}$, denoted by $\bar{y} \in \operatorname{Min}(A, \mathcal{C})$ $[\bar{y} \in \operatorname{Max}(A, \mathcal{C})]$, if and only if

$$
(\mathrm{A}-\overline{\mathrm{y}}) \cap(-\mathcal{C})=\{0\}[(\mathrm{A}-\overline{\mathrm{y}}) \cap \mathcal{C}=\{0\}],
$$

i.e., there is no $y \in A$ such $y \leqslant e ~ \bar{y}[\bar{y} \leqslant \mathcal{e} y]$. If $\operatorname{int}(\mathcal{C}) \neq \emptyset$, then $\bar{y} \in A$ is said to be a weak minimizer of $A$ with respect to $\mathcal{C}$, if

$$
(A-\bar{y}) \cap(-\operatorname{int}(\mathcal{C}))=\emptyset .
$$

As in the literature, if $\bar{y} \in \operatorname{Min}(A, C)[\bar{y} \in \operatorname{Max}(A, C)], \bar{y}$ is also called an efficient point of $A$ with respect to $\mathcal{C}[-\mathcal{C}]$. In this paper, since we consider minimization in a primal problem and maximization in a dual problem, we adopt the terminology minimizer and maximizer. Let $\Theta$ be a base of $\mathcal{C}$. Then $0 \notin \operatorname{cl}(\Theta)$. Employing the Hahn-Banach separation theorem, we get there exists $y_{\Theta}^{*} \in \mathrm{Y}^{*}$ such that

$$
\inf \left\{\left\langle y_{\Theta}^{*}, \theta\right\rangle: \theta \in \Theta\right\}=\alpha>0 \text {. }
$$

Let

$$
V_{\Theta}:=\left\{y \in Y:\left|\left\langle y_{\Theta}^{*}, y\right\rangle\right|<\frac{\alpha}{2}\right\} .
$$

It has been pointed out in [19] that $V_{\Theta}$ is a neighborhood of 0 in $Y$, and for each convex neighborhood $V$ of 0 in $Y$ with $V \subset V_{\Theta}, \Theta+V$ is convex and cone $(\Theta+V)$ is a convex pointed cone.

Definition 2.2 ([19]). Let $\Theta$ be a base of $\mathcal{C}, A$ be a nonempty subset of $Y$ and $\bar{y} \in A$.

(i) $\bar{y}$ is said to be a Henig proper minimizer [maximizer] with respect to $\Theta$, written as $\bar{y} \in \operatorname{HeMin}(A, \Theta)$ $[\bar{y} \in \operatorname{HeMax}(A, \Theta)]$, if there is a convex neighborhood $V$ of 0 in $Y$ with $V \subset V_{\Theta}$ such that

$$
\operatorname{cl}(\operatorname{cone}(A-\bar{y})) \cap(-\operatorname{cone}(\Theta+V))=\{0\}[\operatorname{cl}(\operatorname{cone}(A-\bar{y})) \cap \operatorname{cone}(\Theta+V)=\{0\}] .
$$

(ii) $\bar{y}$ is said to be a super minimizer [maximizer] with respect to $\mathcal{C}$, written as $\bar{y} \in \operatorname{SuMin}(A, \mathcal{C})[\bar{y} \in$ $\operatorname{SuMax}(A, C)]$, if for each neighborhood $V$ of 0 in $Y$, there exits a neighborhood $U$ of 0 in $Y$ such that

$$
\operatorname{cl}(\operatorname{cone}(A-\bar{y})) \cap(U-\mathcal{C}) \subset \mathrm{V}[\operatorname{cl}(\operatorname{cone}(A-\bar{y})) \cap(\mathcal{C}-\mathrm{U}) \subset \mathrm{V}] .
$$

Lemma 2.3 ([19]). Let $\Theta$ be a bounded base of $\mathcal{C}$ and $A$ be a nonempty subset of $Y$. Then

$$
\operatorname{SuMin}(A, \mathcal{C})=\operatorname{SuMin}(A+\mathcal{C}, \mathcal{C})=\operatorname{HeMin}(A+\mathcal{C}, \Theta)=\operatorname{HeMin}(A, \Theta) \subset \operatorname{Min}(A, \mathcal{C}) .
$$

Remark 2.4. Let $\Theta$ be a bounded base of $\mathcal{C}, A$ be a nonempty subset of $Y$, and $\bar{y} \in A$.

(i) By Lemma 2.3 and Definition 2.2, we get that $\bar{y} \in \operatorname{SuMin}(A, \mathcal{C})$ if and only if there is a convex neighborhood $\mathrm{V}$ of 0 in $\mathrm{Y}$ with $\mathrm{V} \subset \mathrm{V}_{\Theta}$ such that

$$
\mathrm{cl}(\operatorname{cone}(A-\bar{y})) \cap(-\operatorname{cone}(\Theta+\mathrm{V}))=\{0\} .
$$

(ii) By a similar argument as that of [19, Lemmas 2.1-2.3], we can obtain the following conclusion:

$$
\operatorname{SuMax}(A, \mathcal{C})=\operatorname{SuMax}(A+\mathcal{C}, \mathcal{C})=\operatorname{HeMax}(A+\mathcal{C}, \Theta)=\operatorname{HeMax}(A, \Theta) \subset \operatorname{Max}(A, \mathcal{C}) .
$$

Let $\mathrm{F}: \mathrm{X} \rightarrow 2^{\mathrm{Y}}$ be a set-valued mapping. The set

$$
\operatorname{im}(F):=\bigcup\{F(x): x \in X\},
$$

is called the image of $F$. The set

$$
\operatorname{graph}(F):=\{(x, y) \in X \times Y: y \in F(x)\},
$$

is called the graph of the mapping F. Lin [11] defined the subgradient for a set-valued mapping in the sense of weak efficiency. Analogously, we extend this concept to super efficiency. 
Definition 2.5. Let $F: X \rightarrow 2^{Y}$ be a set-valued mapping and $(x, y) \in \operatorname{graph}(F)$. An operator $T \in L(X, Y)$ is said to be a super $\mathcal{C}$-subgradient of $F$ at $(\bar{x}, \bar{y})$, if

$$
y-T(x) \in \operatorname{SuMin}(\operatorname{im}(F-T), \mathcal{C}) .
$$

The set of all super subgradients of $F$ at $(x, y)$ is denoted by $\partial_{s u} F(\bar{x}, \bar{y})$.

Remark 2.6. In Definition 2.5, if $Y=\mathbb{R}$ (the set of real numbers) and $\mathcal{C}=\mathbb{R}_{+}$, we write $\partial$ instead of $\partial_{\mathrm{Su}}$. In this case, $T \in \partial_{S u} F(\bar{x}, \bar{y})$ means that $T \in X^{*}$ and

$$
y^{\prime}-y \geqslant T\left(x^{\prime}-x\right), \quad \forall x^{\prime} \in \operatorname{dom}(F), \forall y^{\prime} \in F\left(x^{\prime}\right),
$$

where $\operatorname{dom}(F):=\{x \in X: F(x) \neq \emptyset\}$.

Now, let us recall the notion of nearly subconvexlikeness for set-valued mappings defined in [14, 22].

Definition $2.7([14,22])$. A set-valued mapping $\mathrm{F}: \mathrm{X} \rightarrow 2^{\mathrm{Y}}$ is called to be nearly $\mathcal{C}$-subconvexlike on $\mathrm{X}$ if $\mathrm{cl}($ cone $(\mathrm{im}(\mathrm{F})+\mathrm{C}))$ is convex.

Given two set-valued mappings $F: X \rightarrow 2^{Y}$ and $G: X \rightarrow 2^{Z}$, let

$$
H(x)=(F(x), G(x)), \quad x \in X .
$$

The product $\mathrm{F} \times \mathrm{G}$ is called nearly $\mathcal{C} \times \mathcal{K}$-subconvexlike on $\mathrm{X}$ if $\mathrm{H}$ is nearly $\mathcal{C} \times \mathcal{K}$-subconvexlike on $\mathrm{X}$.

Lemma 2.8 ([19]). If $\mathrm{F} \times \mathrm{G}$ is nearly $\mathrm{C} \times \mathcal{K}$-subconvexlike on $\mathrm{X}$, then for each $\mathrm{T} \in \mathrm{L}_{+}(\mathcal{K}, \mathcal{C}), \mathrm{F}+\mathrm{T} \circ \mathrm{G}$ is nearly $\mathcal{C}$-subconvexlike on $\mathrm{X}$, where $\mathrm{T} \circ \mathrm{G}$ is defined by

$$
\mathrm{T} \circ \mathrm{G}(\mathrm{x})=\bigcup\{\mathrm{T}(z): z \in \mathrm{G}(\mathrm{x})\}, \quad \forall x \in \mathrm{X} .
$$

Assuming that $F: X \rightarrow 2^{Y}$ and $G: X \rightarrow 2^{Z}$ are set-valued mappings, we consider the following set-valued optimization problem (SOP):

$$
\text { (SOP) } \begin{cases}\mathcal{C}-\text { minimize } & \mathrm{F}(\mathrm{x}), \\ \text { subject to } & \mathrm{G}(\mathrm{x}) \cap(-\mathcal{K}) \neq \emptyset, \\ & x \in \mathrm{X} .\end{cases}
$$

The set of feasible solutions of (SOP) is denoted by $\Omega$, that is

$$
\Omega=\{x \in X: G(x) \cap(-\mathcal{K}) \neq \emptyset\} .
$$

For convention, we use

$$
F(\Omega)=\bigcup\{F(x): x \in \Omega\} .
$$

Definition 2.9 ([19]). Let $(\bar{x}, \bar{y}) \in \operatorname{graph}(F)$.

(i) $(\bar{x}, \bar{y})$ is said to be a minimizer of (SOP), if $\bar{x} \in \Omega$ and $\bar{y} \in F(\bar{x}) \cap \operatorname{Min}(F(\Omega), \mathcal{C})$.

(ii) $(\bar{x}, \bar{y})$ is said to be a super minimizer of (SOP), if $\bar{x} \in \Omega$ and $\bar{y} \in F(\bar{x}) \cap \operatorname{SuMin}(F(\Omega), \mathcal{C})$.

Lemma 2.10 ([21]). Let $\Theta$ be a bounded base of $\mathcal{C}$. Let $\bar{x} \in \Omega, \bar{y} \in \mathrm{F}(\bar{x})$ and $\bar{z} \in \mathrm{G}(\bar{x}) \cap(-\mathcal{K})$. Suppose that $(\bar{x}, \bar{y})$ is a super minimizer of $(S O P),(F-\bar{y}, G)$ is nearly $\mathcal{C} \times \mathcal{K}$-subconvexlike on $\mathrm{X}$ and there exists an $\mathrm{x}^{\prime} \in \mathrm{X}$ such that $\mathrm{G}\left(\mathrm{x}^{\prime}\right) \cap(-\operatorname{int}(\mathcal{K})) \neq \emptyset$. Then

(i) there exist $\bar{y}^{*} \in \Theta^{\text {st }}$ and $\bar{z}^{*} \in \mathcal{K}^{+}$such that $\left\langle\bar{z}^{*}, \bar{z}\right\rangle=0$ and

$$
\left\langle\bar{y}^{*}, y\right\rangle+\left\langle\bar{z}^{*}, z\right\rangle \geqslant\left\langle\bar{y}^{*}, \bar{y}\right\rangle, \quad \forall(y, z) \in \operatorname{im}(F \times G) ;
$$


(ii) there exists $\overline{\mathrm{T}} \in \mathrm{L}_{+}(\mathcal{K}, \mathcal{C})$ such that $\overline{\mathrm{T}}(\bar{z})=0$ and $(\overline{\mathrm{x}}, \overline{\mathrm{y}})$ is a super minimizer of the following unconstrained optimization problem:

$$
\begin{cases}\mathrm{e}-\text { minimize } & \mathrm{F}(\mathrm{x})+\overline{\mathrm{T}} \circ \mathrm{G}(\mathrm{x}), \\ \text { subject to } & \mathrm{x} \in \mathrm{X} .\end{cases}
$$

Theorem 2.11. Let $\Theta$ be a bounded base of $\mathcal{C}$. Let $\bar{x} \in \Omega, \bar{y} \in \mathrm{F}(\bar{x})$ and $\bar{z} \in \mathrm{G}(\bar{x}) \cap(-\mathcal{K})$. Suppose that $(\bar{x}, \bar{y})$ is a super minimizer of $(S O P),(F-\bar{y}, G)$ is nearly $\mathcal{C} \times \mathcal{K}$-subconvexlike on $\mathrm{X}$ and there exists an $x^{\prime} \in X$ such that $\mathrm{G}\left(\mathrm{x}^{\prime}\right) \cap(-\operatorname{int}(\mathcal{K})) \neq \emptyset$. Then

(i) there exist $\bar{y}^{*} \in \Theta^{\text {st }}$ and $\bar{z}^{*} \in \mathcal{K}^{+}$such that $\left\langle\bar{z}^{*}, \bar{z}\right\rangle=0$ and

$$
0 \in \partial\left(\bar{y}^{*} \circ F+\bar{z}^{*} \circ G\right)\left(\bar{x},\left\langle\bar{y}^{*}, \bar{y}\right\rangle+\left\langle\bar{z}^{*}, \bar{z}\right\rangle\right) ;
$$

(ii) there exists $\overline{\mathrm{T}} \in \mathrm{L}_{+}(\mathcal{K}, \mathcal{C})$ such that $\overline{\mathrm{T}}(\bar{z})=0$ and

$$
0 \in \partial_{\mathrm{Su}}(\mathrm{F}+\overline{\mathrm{T}} \circ \mathrm{G})(\bar{x}, \bar{y}+\overline{\mathrm{T}}(\bar{z})) .
$$

Proof. From Lemma 2.10 (i), we obtain that there exists $\left(\bar{y}^{*}, \bar{z}^{*}\right) \in \Theta^{\text {st }} \times \mathcal{K}^{+}$such that $\left\langle\bar{z}^{*}, \bar{z}\right\rangle=0$ and

$$
\left\langle\bar{y}^{*}, y\right\rangle+\left\langle\bar{z}^{*}, z\right\rangle \geqslant\left\langle\bar{y}^{*}, \bar{y}\right\rangle=\left\langle\bar{y}^{*}, \bar{y}\right\rangle+\left\langle\bar{z}^{*}, \bar{z}\right\rangle, \quad \forall(y, z) \in \operatorname{im}(F \times G),
$$

which means (2.2) holds. This proves statement (i).

It yields from Lemma 2.10 (ii) that there exists $\bar{T} \in L_{+}(\mathcal{K}, \mathcal{C})$ such that $\bar{T}(\bar{z})=0$ and $(\bar{x}, \bar{y})$ is a super minimizer of the problem:

$$
\begin{cases}\mathcal{C}-\text { minimize } & F(x)+\overline{\mathrm{T}} \circ \mathrm{G}(x), \\ \text { subject to } & x \in X .\end{cases}
$$

This means that for any neighbourhood $V$ of 0 in $Y$, there exists a neighbourhood of $U$ of 0 such that

$$
\operatorname{cl}(\text { cone }(\operatorname{im}(T+\bar{T} \circ G)-\bar{y})) \cap(U-\mathcal{C}) \subset V .
$$

Since $\overline{\mathbf{T}}(\bar{z})=0$, we get that

$$
\mathrm{cl}(\text { cone }(\operatorname{im}(\mathrm{T}+\overline{\mathrm{T}} \circ \mathrm{G})-(\overline{\mathrm{y}}+\overline{\mathrm{T}}(\bar{z})))) \cap(\mathrm{U}-\mathrm{C}) \subset \mathrm{V},
$$

which shows that (2.3) holds. This proves statement (ii).

Remark 2.12. A classical approach to construct $\overline{\mathrm{T}}$ in Lemma 2.10 or Theorem 2.11 is as follows (see the proof of $\left[19,21\right.$, Theorem 4.1]): Since $\bar{y}^{*} \in \Theta^{s t}=\operatorname{int}\left(\mathcal{C}^{+}\right)$, we can choose $\bar{c} \in \mathcal{C}$ such that $\left\langle\bar{y}^{*}, \bar{c}\right\rangle=1$, then the operator $\overline{\mathrm{T}}: Z \rightarrow Y$, defined by $\overline{\mathrm{T}}(z)=\left\langle\overline{\mathrm{y}}^{*}, z\right\rangle \overline{\mathrm{c}}$, for $z \in Z$, is the desired one.

\section{Strong duality}

In this section, we formulate four dual problems of (SOP) and establish several duality theorems. We begin with the classical Lagrange dual problem.

For each $T \in \mathrm{L}_{+}(\mathcal{K}, \mathcal{C})$, the superdual map for (SOP) (see $\left.[12,19]\right)$ is defined by

$$
\Psi(\mathrm{T}):=\operatorname{SuMin}(\operatorname{im}(\mathrm{F}+\overline{\mathrm{T}} \circ \mathrm{G}), \mathcal{C}) .
$$

Then the Lagrange dual problem of (SOP) is formulated as the following problem (see $[12,19])$

$$
\text { (LD) } \quad \begin{cases}\mathcal{C}-\text { maximize } & \Psi(\mathrm{T}) \\ \text { subject to } & \mathrm{T} \in \mathrm{L}_{+}(\mathcal{K}, \mathcal{C}) .\end{cases}
$$

In the next subsection, we will give conditions under which strong duality holds in the sense that, starting from a super minimizer of the primal problem, we can construct a super maximizer of the dual problems. 


\subsection{Lagrange-Wolfe strong duality}

For each $T \in L_{+}(\mathcal{K}, \mathcal{C})$, observing that $y \in \Psi(T)$ if and only if there exist $\xi \in X, u \in F(\xi)$ and $v \in G(\xi)$ such that

$$
y=u+T(v) \in S E(\operatorname{im}(F+\bar{T} \circ G), \mathcal{C}),
$$

or, equivalently, $y=u+T(v)$ and

$$
0 \in \partial_{S u}(F+\bar{T} \circ G)(\xi, u+T(v)) .
$$

Based upon the above observation, we can rewrite the Lagrange dual problem in the form similar to the Wolfe dual problem (see [18]), which is formulated as follows:

$$
\begin{array}{ll}
\text { (LWD) maximize } & u+T(v), \\
\text { subject to } & (u, v) \in F(\xi) \times G(\xi), \quad \xi \in X, \\
& 0 \in \partial_{S u}(F+T \circ G)(\xi, u+T(v)), \\
& T \in L_{+}(\mathcal{C}, \mathcal{K}) .
\end{array}
$$

Denote by $Q_{1}$ the set of all feasible points of (LWD), i.e., the set of points $(\xi, u, v, T) \in X \times Y \times Z \times$ $L(Z, Y)$ satisfying (3.1), (3.2), (3.3). Let $S_{1}$ be the set of all points $u+T(v)$ such that there exists $\xi \in X$ with $(\xi, \mathrm{u}, v, \mathrm{~T}) \in \mathrm{Q}_{1}$.

Definition 3.1. If $(\xi, u, v, T) \in Q_{1}$ and $u+T(v) \in \operatorname{SuMax}\left(S_{1}, \mathcal{C}\right)$, then we say that $(\xi, u, v, T)$ is a super maximizer of problem (LWD).

Theorem 3.2 (Weak Duality). Let $\Theta$ be a bounded base of $\mathcal{C}$. If $x \in \Omega$ and $(\xi, \mathrm{u}, v, \mathrm{~T}) \in \mathrm{Q}_{1}$, then there exists a convex open neighborhood $\mathrm{V}$ of 0 in $\mathrm{Y}$ with $\mathrm{V} \subset \mathrm{V}_{\Theta}$ such that

$$
\mathrm{cl}(\operatorname{cone}(\mathrm{F}(\mathrm{x})-(\mathrm{u}+\mathrm{T}(\mathrm{v})))) \cap(-\operatorname{cone}(\Theta+\mathrm{V}))=\{0\} .
$$

Proof. Since $x \in \Omega$, it holds that $\mathrm{G}(x) \cap(-\mathcal{K}) \neq \emptyset$. So, we can take a point $v^{\prime} \in \mathrm{G}(x) \cap(-\mathcal{K})$ such that

$$
-\mathrm{T}\left(v^{\prime}\right) \in \mathrm{T}(\mathcal{K}) \subset \mathcal{e} \text {. }
$$

Hence,

$$
\begin{aligned}
\mathrm{F}(\mathrm{x})-(\mathrm{u}+\mathrm{T}(v)) & =\mathrm{F}(\mathrm{x})+\mathrm{T}\left(v^{\prime}\right)-(\mathrm{u}+\mathrm{T}(v))-\mathrm{T}\left(v^{\prime}\right) \\
& \subset \mathrm{F}(\mathrm{x})+\mathrm{T}\left(v^{\prime}\right)-(\mathrm{u}+\mathrm{T}(v))+\mathcal{C} \\
& \subset \mathrm{F}(\mathrm{x})+\mathrm{T} \circ \mathrm{G}(\mathrm{x})-(\mathrm{u}+\mathrm{T}(v))+\mathcal{C} \\
& \subset \mathrm{im}(\mathrm{F}+\mathrm{T} \circ \mathrm{G})-(\mathrm{u}+\mathrm{T}(v))+\mathcal{C} .
\end{aligned}
$$

On the other hand, from (3.2) and Remark 2.4, we obtain that there exists a convex open neighborhood $\mathrm{V}$ of 0 in $\mathrm{Y}$ with $\mathrm{V} \subset \mathrm{V}_{\Theta}$ (defined as in (2.1)) such that

$$
\mathrm{cl}(\operatorname{cone}(\mathrm{im}(\mathrm{F}+\mathrm{T} \circ \mathrm{G})-(\mathrm{u}+\mathrm{T}(\mathrm{v})))) \cap(-\operatorname{cone}(\mathrm{V}+\Theta))=\{0\} .
$$

It follows from Lemma 2.3 that

$$
\mathrm{cl}(\operatorname{cone}(\mathrm{im}(\mathrm{F}+\mathrm{T} \circ \mathrm{G})-(\mathrm{u}+\mathrm{T}(\mathrm{v}))+\mathrm{C})) \cap(-\operatorname{cone}(\mathrm{V}+\Theta))=\{0\} .
$$

Thus, from (3.5), we infer that (3.4) holds as desired.

Remark 3.3. In Theorem 3.2, it follows from (3.4) and Lemma 2.3 that $u+T(v) \in \operatorname{Min}(F(x), \mathcal{C})$. This leads to

$$
(\mathrm{F}(\mathrm{x})-(\mathrm{u}+\mathrm{T}(v))) \cap(-\mathrm{C} \backslash\{0\})=\emptyset .
$$

So, (3.4) means that

$$
y \not e \mathrm{u}+\mathrm{T}(v), \quad \forall \mathrm{y} \in \mathrm{F}(\mathrm{x}),
$$

which is the general weak duality in the literatures $[12,19]$. 
Theorem 3.4 (Strong Duality). Let $\mathcal{C}$ have a bounded base $\Theta$. Let $\bar{x} \in \Omega, \bar{y} \in \mathrm{F}(\bar{x})$ and $\bar{z} \in \mathrm{G}(\bar{x}) \cap(-\mathcal{K})$. Suppose that $(\bar{x}, \bar{y})$ is a super minimizer of $(S O P),(F-\bar{y}, G)$ is nearly $\mathcal{C} \times \mathcal{K}$-subconvexlike on $\mathrm{X}$, and there exists an $\mathrm{x}^{\prime} \in \mathrm{X}$ such that $\mathrm{G}\left(\mathrm{x}^{\prime}\right) \cap(-\operatorname{int}(\mathcal{K})) \neq \emptyset$. Then there exists $\overline{\mathrm{T}} \in \mathrm{L}_{+}(\mathcal{K}, \mathcal{C})$ such that $\overline{\mathrm{T}}(\bar{z})=0,(\overline{\mathrm{x}}, \overline{\mathrm{y}}, \bar{z}, \overline{\mathrm{T}})$ is a super maximizer of (LWD), and the corresponding objective values of (SOP) and (LWD) are equal.

Proof. It yields from Theorem 2.11 that there exists $\bar{T} \in L_{+}(Z, Y)$ such that $\bar{T}(\bar{z})=0$ and $(\bar{x}, \bar{y}, \bar{z}, \bar{T}) \in Q_{1}$. It remains to prove that $\bar{y}=\bar{y}+\bar{T}(\bar{z}) \in \operatorname{SuMax}\left(S_{1}, \mathcal{C}\right)=\operatorname{HeMax}\left(S_{1}, \Theta\right)$ (since $\mathcal{C}$ have a bounded base $\Theta$, see Remark 2.4). Suppose that this is not true. Then for each convex neighbourhood $V$ of 0 in $Y$ with $V \subset V_{\Theta}$, the following holds

$$
\operatorname{cl}\left(\operatorname{cone}\left(\mathrm{S}_{1}-(\overline{\mathrm{y}}+\mathrm{T}(\bar{z}))\right)\right) \cap \operatorname{cone}(\Theta+\mathrm{V}) \neq\{0\} .
$$

Thus, there exist $\mathrm{b}_{0} \in \operatorname{cone}(\Theta+\mathrm{V}) \backslash\{0\}, \lambda>0$, and $\hat{u}+\mathrm{T}(\hat{v}) \in \mathrm{S}_{1}$ such that

$$
b_{0}=\lambda(\hat{u}+T(\hat{v})-\bar{y}),
$$

or, equivalently,

$$
-\mathrm{b}_{0}=\lambda(\overline{\mathrm{y}}-(\hat{\mathrm{u}}+\mathrm{T}(\hat{v}))) \in \operatorname{cl}(\operatorname{cone}(\mathrm{F}(\overline{\mathrm{x}})-(\hat{\mathrm{u}}+\mathrm{T}(\hat{v})))) .
$$

This indicates that

$$
(-\operatorname{cone}(\Theta+V)) \cap \operatorname{cl}(\operatorname{cone}(F(\bar{x})-(\hat{u}+T(\hat{v})))) \neq\{0\},
$$

a contradiction to the weak duality property (3.4) with $x=\bar{x}$.

Remark 3.5. It is well-known that if a set-valued mapping is nearly convexlike, then it is nearly subconvexlike. Mehra [12] and Xia and Qiu [19] established duality theorems between (SOP) and (LD) (Lagrange dual problem) under the assumptions that the involved set-valued mappings are nearly convexlike and nearly subconvexlike, respectively. Their strong duality between (SOP) and (LD) was presented in the sense that a super minimizer of (SOP) is a maximizer of (LD) (see [12, 19, Theorem 5.2]). However, we are interested in the problem that a super minimizer of (SOP) is a super maximizer of (LD). So, Theorem 3.4 is an improvement of the corresponding results in $[12,19]$.

\subsection{Mond-Weir strong duality}

This subsection is dedicated to construct another dual problem based on the idea of Mond-Weir (see [17]), called the Mond-Weir duality problem (MWD). A strong duality result between (SOP) and (MWD) will be established.

The following problem is named the Mond-Weir dual problem of (SOP) and is denoted by (MWD):

$$
\begin{array}{ll}
\text { (MWD) maximize } & \mathrm{u} \\
\text { subject to } & (\mathrm{u}, v) \in \mathrm{F}(\xi) \times \mathrm{G}(\xi), \\
& 0 \in \partial_{\mathrm{Su}}(\mathrm{F}+\mathrm{T} \circ \mathrm{G})(\xi, \mathrm{u}+\mathrm{T}(v)), \\
& \mathrm{T} \in \mathrm{L}_{+}(\mathcal{K}, \mathcal{C}) \\
& \mathrm{T}(v) \in \mathcal{C} .
\end{array}
$$

Denote by $\mathrm{Q}_{2}$ the set of all feasible points of (MWD), i.e., the set of points $(\xi, u, v, T) \in X \times Y \times Z \times L(Z, Y)$ satisfying (3.6), (3.7), (3.8), and (3.9). Let $S_{2}$ be the set of all points $u$ such that there exists $(\xi, v, T) \in$ $\mathrm{X} \times \mathrm{Z} \times \mathrm{L}(\mathrm{Z}, \mathrm{Y})$ with $(\xi, \mathrm{u}, v, \mathrm{~T}) \in \mathrm{Q}_{2}$.

Definition 3.6. If $(\xi, u, v, T) \in \mathrm{Q}_{2}$ and $u \in \operatorname{SuMax}\left(S_{2}, \mathcal{C}\right)$, then we say that $(\xi, u, v, T)$ is a super maximizer of problem (MWD).

Lemma 3.7. It holds that $\mathrm{Q}_{2} \subset \mathrm{Q}_{1}$ and $\mathrm{S}_{2} \subset \mathrm{S}_{1}-\mathrm{C}$.

Proof. According to the definitions of $\mathrm{Q}_{1}$ and $\mathrm{Q}_{2}$, it is obvious that $\mathrm{Q}_{2} \subset \mathrm{Q}_{1}$. Let $\mathrm{u} \in \mathrm{S}_{2}$. Then there exists 
$(\xi, v, T) \in X \times Z \times L(Z, Y)$ such that $(\xi, u, v, T) \in Q_{2} \subset Q_{1}$. We get that

$$
\begin{aligned}
u & =u+T(v)-T(v) \\
& \in S_{1}-T(v) \\
& \subset S_{1}-\mathcal{C} .
\end{aligned}
$$

Thus, $u \in S_{1}-\mathcal{C}$. This completes the proof of $S_{2} \subset S_{1}-\mathcal{C}$.

Theorem 3.8 (Weak Duality). Let $\Theta$ be a bounded base of $\mathcal{C}$. If $x \in \Omega$ and $(\xi, \mathrm{u}, v, \mathrm{~T}) \in \mathrm{Q}_{2}$, then there exists a convex open neighbourhood $\mathrm{V}$ of 0 in $\mathrm{Y}$ with $\mathrm{V} \subset \mathrm{V}_{\Theta}$ such that

$$
\operatorname{cl}(\operatorname{cone}(F(x)-u)) \cap(-\operatorname{cone}(\Theta+V))=\{0\} .
$$

Proof. From Lemma 3.7, we obtain that $\mathrm{Q}_{2} \subset \mathrm{Q}_{1}$. Again, we get from Theorem 3.2 that there exists a convex open neighbourhood $U$ of 0 in $Y$ such that

$$
\operatorname{cl}(\operatorname{cone}(\mathrm{F}(\mathrm{x})-(\mathrm{u}+\mathrm{T}(\mathrm{v})))) \cap(-\operatorname{cone}(\Theta+\mathrm{V}))=\{0\},
$$

which means $u+T(v) \in \operatorname{SuMin}(F(x), \mathcal{C})$. It follows from Lemma 2.3 that

$$
\operatorname{cl}(\operatorname{cone}(\mathrm{F}(\mathrm{x})-(\mathrm{u}+\mathrm{T}(v))+\mathcal{C})) \cap(-\operatorname{cone}(\Theta+\mathrm{V}))=\{0\} .
$$

On the other hand, it yields from (3.9) that

$$
\begin{aligned}
\mathrm{F}(\mathrm{x})-\mathrm{u} & =\mathrm{F}(\mathrm{x})-(\mathrm{u}+\mathrm{T}(v))+\mathrm{T}(v) \\
& \subset \mathrm{F}(\mathrm{x})-(\mathrm{u}+\mathrm{T}(v))+\mathcal{C} .
\end{aligned}
$$

By combining the above inequality with (3.11), it yields (3.10) as required.

In order to formulate the strong duality between (SOP) and (MWD), we need to prove the following lemma.

Lemma 3.9. Let $\Theta$ be a bounded base of $\mathcal{C}$. If $(\bar{\xi}, \bar{u}, \bar{v}, \overline{\mathbf{T}})$ is a super maximizer of (LWD) and $\overline{\mathbf{T}}(\bar{v})=0$, then $(\bar{\xi}, \bar{u}, \bar{v}, \bar{T})$ is a super maximizer of (MWD) and the corresponding objective values of both problems are equal.

Proof. Because $(\bar{\xi}, \bar{u}, \bar{v}, \bar{T})$ is a super maximizer of (LWD), it follows from the definition of $S_{1}$ and Remark 2.4 that there exists a convex open neighbourhood $V$ of 0 in $Y$ with $V \subset V_{\Theta}$ such that

$$
\operatorname{cl}\left(\operatorname{cone}\left(S_{1}-(\bar{u}+\bar{T}(\bar{v}))\right)\right) \cap \operatorname{cone}(\Theta+V)=\{0\} .
$$

Therefore, we get from Remark 2.4 that

$$
\operatorname{cl}\left[\operatorname{cone}\left(S_{1}-(\bar{u}+\bar{T}(\bar{v}))-\mathcal{C}\right)\right] \cap \operatorname{cone}(\Theta+V)=\{0\} .
$$

On the other hand, according to Lemma 3.7, we have $S_{2} \subset S_{1}-\mathcal{C}$. We derive from $\bar{T}(\bar{v})=0$ that

$$
\mathrm{S}_{2}-\overline{\mathrm{u}} \subset \mathrm{S}_{1}-(\overline{\mathrm{u}}+\overline{\mathrm{T}}(\bar{v}))-\mathcal{C} .
$$

In view of (3.12) and (3.13), it is clear that

$$
\operatorname{cl}\left(\operatorname{cone}\left(S_{2}-\bar{u}\right)\right) \cap \operatorname{cone}(\Theta+V)=\{0\},
$$

which is the desired result. 
Theorem 3.10 (Strong Duality). Let $\mathcal{C}$ have a bounded base $\Theta$. Let $\bar{x} \in \Omega, \bar{y} \in \mathrm{F}(\bar{x})$, and $\bar{z} \in \mathrm{G}(\bar{x}) \cap(-\mathcal{K})$. Suppose that $(\bar{x}, \bar{y})$ is a super minimizer of $(S O P),(F-\bar{y}, G)$ is nearly $\mathcal{C} \times \mathcal{K}$-subconvexlike on $\mathrm{X}$, and there exists an $\mathrm{x}^{\prime} \in \mathrm{X}$ such that $\mathrm{G}\left(\mathrm{x}^{\prime}\right) \cap(-\operatorname{int}(\mathcal{K})) \neq \emptyset$. Then there exists $\overline{\mathrm{T}} \in \mathrm{L}_{+}(\mathcal{K}, \mathcal{C})$ such that $\overline{\mathrm{T}}(\bar{z})=0,(\overline{\mathrm{x}}, \overline{\mathrm{y}}, \bar{z}, \overline{\mathrm{T}})$ is a super maximizer of (MWD), and the corresponding objective values of (SOP) and (MWD) are equal.

Proof. It follows from Theorem 2.11 and Lemma 3.7 that there exists $\bar{T} \in L_{+}(\mathcal{K}, \mathcal{C})$ such that $\bar{T}(\bar{z})=0$ and $(\bar{x}, \bar{y}, \bar{z}, \overline{\mathrm{T}}) \in \mathrm{Q}_{2} \subset \mathrm{Q}_{1}$. Hence, we get from the strong duality Theorem 3.4 between (SOP) and (LWD) that $(\bar{x}, \bar{y}, \bar{z}, \bar{T})$ is a super maximizer of (LWD) and the corresponding objective values of (SOP) and (LWD) are equal. It yields from Lemma 3.9 that $(\bar{x}, \bar{y}, \bar{z}, \bar{T})$ is also a super maximizer of (MWD) and the corresponding objective values of (LWD) and (MWD) are equal. So, we infer that there exists $\bar{T} \in L_{+}(Z, Y)$ such that $\bar{T}(\bar{z})=0,(\bar{x}, \bar{y}, \bar{z}, \bar{T})$ is a super maximizer of (MWD), and the corresponding objective values of (SOP) and (MWD) are equal.

\subsection{Mixed Lagrange-Wolfe strong duality}

In this subsection, let $\bar{c} \in \mathcal{C} \backslash\{0\}$ be a fixed point and consider the following problem (MLWD), termed the mixed Lagrange-Wolfe dual problem of (SOP):

$$
\begin{array}{ll}
\text { (MLWD) maximize } & u+\left\langle z^{*}, v\right\rangle \overline{\mathrm{c}} \\
\text { subject to } & (\mathrm{u}, v) \in \mathrm{F}(\xi) \times \mathrm{G}(\xi), \xi \in X, \\
& \left(\mathrm{y}^{*}, z^{*}\right) \in \mathrm{B}^{\mathrm{st}} \times \mathcal{K}^{+}, \\
& 0 \in \partial\left(\mathrm{y}^{*} \circ \mathrm{F}+z^{*} \circ \mathrm{G}\right)\left(\xi,\left\langle\mathrm{y}^{*}, \mathrm{u}\right\rangle+\left\langle z^{*}, v\right\rangle\right), \\
& \left\langle\mathrm{y}^{*}, \overline{\mathrm{c}}\right\rangle=1 .
\end{array}
$$

Notice that (3.16) means that

$$
\left\langle y^{*}, y\right\rangle+\left\langle z^{*}, z\right\rangle \geqslant\left\langle y^{*}, u\right\rangle+\left\langle z^{*}, v\right\rangle, \quad \forall(y, z) \in \operatorname{im}(F \times G) .
$$

Denote by $\mathrm{Q}_{3}$ the set of all feasible points of (MLWD), i.e., the set of points $\left(\xi, u, v, y^{*}, z^{*}\right) \in X \times Y \times Z \times$ $\mathrm{Y}^{*} \times \mathrm{Z}^{*}$ satisfying (3.14), (3.15), (3.16), and (3.17). Let

$$
\mathrm{S}_{3}:=\left\{u+\left\langle z^{*}, v\right\rangle \bar{c} \text { : there exists }\left(\xi, y^{*}\right) \text { such that }\left(\xi, u, v, y^{*}, z^{*}\right) \in Q_{3}\right\} .
$$

Definition 3.11. If $\left(\xi, u, v, y^{*}, z^{*}\right) \in \mathrm{Q}_{3}$ and $u+\left\langle z^{*}, v\right\rangle \overline{\mathrm{c}} \in \operatorname{SuMax}\left(S_{3}, \mathcal{C}\right)$, then we say that $\left(\xi, u, v, y^{*}, z^{*}\right)$ is a super maximizer of problem (MLWD).

The following Lemma 3.12 discloses a relationship between $Q_{1}$ and $Q_{3}$.

Lemma 3.12. Let $\Theta$ be a bounded base of $\mathrm{C}$. The following statements hold.

(i) If $\left(\xi, \mathrm{u}, v, \mathrm{y}^{*}, z^{*}\right) \in \mathrm{Q}_{3}$, then $(\xi, \mathrm{u}, v, \mathrm{~T}) \in \mathrm{Q}_{1}$, where $\mathrm{T}$ is defined by $\mathrm{T}(\cdot)=\left\langle z^{*}, \cdot\right\rangle \overline{\mathrm{c}}$.

(ii) Let $(\xi, \mathrm{u}, v, \mathrm{~T}) \in \mathrm{Q}_{1}$ with $\mathrm{T}(v)=0$. If $(\mathrm{F}-\mathrm{u}, \mathrm{G})$ is nearly $\mathcal{C} \times \mathcal{K}$-subconvexlike on $\mathrm{X}$, then there exists $\left(\mathrm{y}^{*}, z^{*}\right) \in \mathrm{Y}^{*} \times \mathrm{Z}^{*}$ such that $\left(\xi, \mathrm{u}, v, \mathrm{y}^{*}, z^{*}\right) \in \mathrm{Q}_{3}$ and $\left\langle\mathrm{y}^{*}, \mathrm{~T}(\cdot)\right\rangle=\left\langle z^{*}, \cdot\right\rangle$.

Proof. The first part of Lemma 3.12 can be derived by the argument used in the proof of [19, Theorem 4.1] (or see Remark 2.12). Let us prove the second part. Since $(F-u, G)$ is nearly $\mathcal{C} \times \mathcal{K}$-subconvexlike on $X$, from Lemma 2.8, we know that $F+T \circ G-u$ is nearly $\mathcal{C}$-subconvexlike on $X$. Thus, we get that $\mathrm{cl}(\operatorname{cone}(\operatorname{im}(F+T \circ G)-u+\mathcal{C}))$ is a convex set. Noticing $T(v)=0$, we obtain that $\operatorname{cl}(\operatorname{cone}(\operatorname{im}(F+T \circ$ $\mathrm{G})-(u+T(v))+\mathcal{C})$ ) is a convex set. Because $\Theta$ is a bounded base of $\mathcal{C}$, it follows from Lemma 2.3 that $\operatorname{SuMin}(\operatorname{im}(F+T \circ G), \mathcal{C})=\operatorname{SuMin}(\operatorname{im}(F+T \circ G)+\mathcal{C}, \mathcal{C})=\operatorname{HeMin}(\operatorname{im}(F+T \circ G), \Theta)$. In view of (3.2), we infer that there exists a convex open neighborhood $V$ of 0 in $Y$ with $V \subset V_{\Theta}$ such that

$$
\operatorname{cl}(\operatorname{cone}(\operatorname{im}(F+T \circ G)-(u+T(v))+\mathcal{C})) \cap(-\operatorname{cone}(V+\Theta))=\{0\},
$$


which implies

$$
\mathrm{cl}(\operatorname{cone}(\mathrm{im}(\mathrm{F}+\mathrm{T} \circ \mathrm{G})-(\mathrm{u}+\mathrm{T}(v))+\mathcal{C})) \cap(-\mathrm{V}-\Theta)=\emptyset .
$$

Hence, by the Hahn-Banach separation theorem, there exists $y^{*} \in Y^{*} \backslash\{0\}$ such that

$$
\left\langle y^{*}, y\right\rangle>\left\langle y^{*}, y^{\prime}\right\rangle \forall y \in \text { cone }(\operatorname{im}(F+T \circ G)-(u+T(v))+\mathcal{C}), y^{\prime} \in-V-\Theta .
$$

Thus, we obtain that

$$
\left\langle y^{*}, y-(u+T(v))+c\right\rangle \geqslant 0, \quad \forall y \in \operatorname{im}(F+T \circ G), \quad \forall x \in \mathcal{C},
$$

and

$$
\left\langle y^{*}, y^{\prime}\right\rangle<0, \quad \forall y^{\prime} \in-V-\Theta .
$$

Furthermore, it implies that $\mathrm{y}^{*} \in \mathrm{B}^{\text {st }}$ due to Lemma 2.1. By replacing $\mathrm{y}^{*}$ by $\mathrm{y}^{*} /\left\langle\mathrm{y}^{*}, \overline{\mathrm{c}}\right\rangle$ if necessary, we may assume that $\left\langle\mathrm{y}^{*}, \overline{\mathrm{c}}\right\rangle=1$. So, (3.17) is satisfied. Defining linear functional $z^{*}$ by $\left\langle z^{*}, \cdot\right\rangle=\left\langle\mathrm{y}^{*}, \mathrm{~T}(\cdot)\right\rangle$, from (3.3) we get $z^{*} \in \mathcal{K}^{+}$. It yields from (3.19) with $c=0$ that (3.18) is fulfilled. So, all conditions (3.14), (3.15), (3.16), and (3.17) are satisfied and hence $\left(\xi, u, v, y^{*}, z^{*}\right) \in Q_{3}$. This completes the proof.

Lemma 3.13. It holds that $S_{3} \subset S_{1}$.

Proof. This is an immediate consequence of Lemma 3.12.

Theorem 3.14 (Weak Duality). Let $\Theta$ be a bounded base of $\mathcal{C}$. If $\mathrm{x} \in \Omega$ and $\left(\xi, \mathrm{u}, v, \mathrm{y}^{*}, z^{*}\right) \in \mathrm{Q}_{3}$, then there exists a convex open neighborhood $\mathrm{V}$ of 0 in $\mathrm{Y}$ with $\mathrm{V} \subset \mathrm{V}_{\Theta}$ such that

$$
\operatorname{cl}\left(\operatorname{cone}\left(\mathrm{F}(\mathrm{x})-\left(\mathrm{u}+\left\langle z^{*}, v\right\rangle \overline{\mathbf{c}}\right)\right)\right) \cap(-\operatorname{cone}(\Theta+\mathrm{V}))=\{0\} .
$$

Proof. From Lemma 3.12, we get that $(\xi, u, v, T) \in \mathrm{Q}_{1}$ with $\mathrm{T}(\cdot)=\left\langle z^{*}, \cdot\right\rangle \bar{c}$. In view of weak duality between (SOP) and (LWD) (see Theorem 3.2), we obtain that there exists a convex open neighborhood $V$ of 0 in $Y$ with $\mathrm{V} \subset \mathrm{V}_{\Theta}$ such that

$$
\mathrm{cl}[\operatorname{cone}(\mathrm{F}(\mathrm{x})-(\mathrm{u}+\mathrm{T}(v)))] \cap(-\operatorname{cone}(\Theta+\mathrm{V}))=\{0\} .
$$

Noticing $T(v)=\left\langle z^{*}, v\right\rangle \bar{c}$, we get the desired conclusion.

Theorem 3.15 (Strong Duality). Let $\mathcal{C}$ have a bounded base $\Theta$. Let $\bar{x} \in \Omega, \bar{y} \in \mathrm{F}(\bar{x})$, and $\bar{z} \in \mathrm{G}(\bar{x}) \cap(-\mathcal{K})$. Suppose that $(\bar{x}, \bar{y})$ is a super minimizer of $(S O P),(F-\bar{y}, G)$ is nearly $\mathcal{C} \times \mathcal{K}$-subconvexlike on $\mathrm{X}$, and there exists an $x^{\prime} \in X$ such that $\mathrm{G}\left(x^{\prime}\right) \cap(-\operatorname{int}(\mathcal{K})) \neq \emptyset$. Then there exists $\left(\bar{y}^{*}, \bar{z}^{*}\right) \in \mathrm{B}^{\text {st }} \times \mathcal{K}^{+}$such $\left\langle\bar{z}^{*}, \bar{z}\right\rangle=0,\left(\bar{x}, \bar{y}, \bar{z}, \bar{y}^{*}, \bar{z}^{*}\right)$ is a super maximizer of (MLWD), and the corresponding objective values of (SOP) and (MLWD) are equal.

Proof. Observing that all conditions of Theorem 3.4 are fulfilled, we get that there exists $\overline{\mathrm{T}} \in \mathrm{L}_{+}(\mathcal{K}, \mathcal{C})$ such that $\bar{T}(\bar{z})=0,(\bar{x}, \bar{y}, \bar{z}, \bar{T})$ is a super maximizer of (LWD) and the objective values of (SOP) and (LWD) are equal to $\bar{y}$. On the other hand, since $(F-\bar{y}, G)$ is nearly $\mathcal{C} \times \mathcal{K}$-subconvexlike on $X$ and $\bar{T}(\bar{z})=0$, it implies from Lemma 2.8 that the map $(F+\bar{T} \circ G)-(\bar{y}+\bar{T}(\bar{z}))$ is nearly C-subconvexlike on $X$. Thus, by Lemma 3.12, it follows that there exists $\left(\bar{y}^{*}, \bar{z}^{*}\right) \in Y^{*} \times Z^{*}$ such that $\left(\bar{x}, \bar{y}, \bar{z}, \bar{y}^{*}, \bar{z}^{*}\right) \in Q_{3}$ and $\bar{z}^{*}$ is defined by

$$
\left\langle\bar{z}^{*}, \cdot\right\rangle=\left\langle\overline{\mathrm{y}}^{*}, \overline{\mathrm{T}}(\cdot)\right\rangle .
$$

Combining the above equality with the fact $\bar{T}(\bar{z})=0$, we derive that $\left\langle\bar{z}^{*}, \bar{z}\right\rangle=0$. Since we have obtained that $\left\langle\bar{z}^{*}, \bar{z}\right\rangle=0, S_{3} \subset S_{1}$ (by Lemma 3.13) and $(\bar{x}, \bar{y}, \bar{z}, \bar{T})$ is a super maximizer of (LWD), it is obvious that $\left(\bar{x}, \bar{y}, \bar{z}, \bar{y}^{*}, \bar{z}^{*}\right)$ is a super maximizer of (MLWD) and the corresponding objective values of (SOP) and (MLWD) are equal to $\bar{y}$. This completes the proof. 


\subsection{Mixed Mond-Weir strong duality}

In this subsection, we work on the following problem (MMWD), named the mixed Mond-Weir dual problem of (SOP):

$$
\begin{aligned}
\text { maximize } & u \\
\text { subject to } & (\mathrm{u}, v) \in \mathrm{F}(\xi) \times \mathrm{G}(\xi), \xi \in \mathrm{X} \\
& \left(\mathrm{y}^{*}, \mathrm{z}^{*}\right) \in \mathrm{B}^{\mathrm{st}} \times \mathcal{K}^{+}, \\
& 0 \in \partial\left(\mathrm{y}^{*} \circ \mathrm{F}+\mathrm{z}^{*} \circ \mathrm{G}\right)\left(\xi,\left\langle\mathrm{y}^{*}, \mathrm{u}\right\rangle+\left\langle\mathrm{z}^{*}, v\right\rangle\right), \\
& \left\langle\mathrm{z}^{*}, v\right\rangle \geqslant 0 .
\end{aligned}
$$

Denote by $\mathrm{Q}_{4}$ the set of all feasible points of (MMWD), i.e., the set of points $\left(\xi, u, v, y^{*}, z^{*}\right) \in X \times Y \times Z \times$ $\mathrm{Y}^{*} \times \mathrm{Z}^{*}$ satisfying (3.20), (3.21), (3.22), and (3.23). Let

$$
S_{4}:=\left\{u \text { : there exists }\left(\xi, v, y^{*}, z^{*}\right) \text { such that }\left(\xi, u, v, y^{*}, z^{*}\right) \in Q_{4}\right\} .
$$

Definition 3.16. If $\left(\xi, u, v, y^{*}, z^{*}\right) \in Q_{4}$ and $u \in \operatorname{SuMax}\left(S_{4}, \mathcal{C}\right)$, then we say that $\left(\xi, u, v, y^{*}, z^{*}\right)$ is a super maximizer of problem (MMWD).

A relationship between $\mathrm{Q}_{2}$ and $\mathrm{Q}_{4}$ is presented in the following lemma.

Lemma 3.17. Let $\Theta$ be a bounded base of $\mathcal{C}$. The following statements hold.

(i) If $\left(\xi, \mathrm{u}, v, \mathrm{y}^{*}, z^{*}\right) \in \mathrm{Q}_{4}$, then there exists $\mathrm{T} \in \mathrm{L}_{+}(\mathrm{Z}, \mathrm{Y})$ such $(\xi, \mathrm{u}, v, \mathrm{~T}) \in \mathrm{Q}_{2}$.

(ii) Let $(\xi, \mathrm{u}, v, \mathrm{~T}) \in \mathrm{Q}_{2}$ with $\mathrm{T}(v)=0$. If $(\mathrm{F}-\mathrm{u}, \mathrm{G})$ is nearly $\mathrm{C} \times \mathcal{K}$-subconvexlike on $\mathrm{X}$, then there exists $\left(y^{*}, z^{*}\right) \in Y^{*} \times Z^{*}$ such that $\left(\xi, u, v, y^{*}, z^{*}\right) \in Q_{4}$.

Proof. (i) We can construct $\mathrm{T}$ by employing the classical approach (see Remark 2.12). That is: taking $\overline{\mathrm{c}} \in \mathcal{C} \backslash\{0\}$ such that $\left\langle\mathrm{y}^{*}, \overline{\mathrm{c}}\right\rangle=1$ and setting $\mathrm{T}(\cdot)=\left\langle z^{*}, \cdot\right\rangle \overline{\mathrm{c}}$, from Theorem 2.11 , we easily see that this $\mathrm{T}$ satisfies (3.7). So, we get $(\xi, \mathrm{u}, v, \mathrm{~T}) \in \mathrm{Q}_{2}$ as desired.

(ii) The point $\left(y^{*}, z^{*}\right)$ can be constructed as in the proof of Lemma 3.12, where $\bar{c}$ is a point of $\mathcal{C}$ with $\left\langle\mathrm{y}^{*}, \overline{\mathrm{c}}\right\rangle=1$ and $z^{*}$ is defined by $\left\langle z^{*}, \cdot\right\rangle=\left\langle\mathrm{y}^{*}, \mathrm{~T}(\cdot)\right\rangle$. It has been pointed out in Lemma 3.12 that $\left(y^{*}, z^{*}\right)$ satisfies (3.22). In addition, noticing that $0=\left\langle y^{*}, T(v)\right\rangle=\left\langle z^{*}, v\right\rangle$, we get that (3.23) is fulfilled. Therefore, we obtain $\left(\xi, u, v, y^{*}, z^{*}\right) \in Q_{4}$.

The following Lemma 3.18 can be derived from Lemma 3.17 directly.

Lemma 3.18. It holds that $S_{4} \subset S_{2}$.

Theorem 3.19 (Weak Duality). Let $\Theta$ be a bounded base of $\mathcal{C}$. If $\mathrm{x} \in \Omega$ and $\left(\xi, \mathrm{u}, v, \mathrm{y}^{*}, z^{*}\right) \in \mathrm{Q}_{4}$, then there exists a convex open neighbourhood $\mathrm{V}$ of 0 in $\mathrm{Y}$ with $\mathrm{V} \subset \mathrm{V}_{\Theta}$ such that

$$
\mathrm{cl}(\operatorname{cone}(\mathrm{F}(\mathrm{x})-\mathrm{u})) \cap(-\operatorname{cone}(\Theta+\mathrm{V}))=\{0\} .
$$

Proof. This can be seen by using Theorem 3.8 and Lemma 3.18.

Lemma 3.20. It holds that $S_{4} \subset S_{3}-\mathcal{C}$.

Proof. Suppose that $u \in S_{4}$. Then there exists $\left(\xi, v, y^{*}, z^{*}\right)$ such that $\left(\xi, u, v, y^{*}, z^{*}\right) \in Q_{4}$. This means (3.20), (3.21), (3.22), and (3.23) are fulfilled. Let $\bar{c}$ be the element appearing in the formulation of (MLWD). By setting $\bar{y}^{*}=y^{*} /\left\langle y^{*}, \bar{c}\right\rangle$ and $\bar{z}^{*}=z^{*} /\left\langle y^{*}, \bar{c}\right\rangle$, it is clear that $\left(\xi, u, v, \bar{y}^{*}, \bar{z}^{*}\right) \in Q_{3}$. Thus, we have $u+\left\langle\bar{z}^{*}, v\right\rangle \bar{c} \in S_{3}$. This implies that

$$
u \in S_{3}-\left\langle\bar{z}^{*}, v\right\rangle \bar{c} \subset S_{3}-\mathcal{C},
$$

due to $\left\langle\bar{z}^{*}, v\right\rangle=\left\langle z^{*}, v\right\rangle /\left\langle\mathrm{y}^{*}, \overline{\mathrm{c}}\right\rangle \geqslant 0$ and $\overline{\mathrm{c}} \in \mathcal{C}$. This completes the proof. 
Theorem 3.21 (Strong Duality). Let $\mathcal{C}$ have a bounded base $\Theta$. Let $\bar{x} \in \Omega, \bar{y} \in \mathrm{F}(\bar{x})$, and $\bar{z} \in \mathrm{G}(\bar{x}) \cap(-\mathcal{K})$. Suppose that $(\bar{x}, \bar{y})$ is a super minimizer of $(S O P),(F-\bar{y}, G)$ is nearly $\mathcal{C} \times \mathcal{K}$-subconvexlike on $\mathrm{X}$, and there exists an $x^{\prime} \in \mathrm{X}$ such that $\mathrm{G}\left(\mathrm{x}^{\prime}\right) \cap(-\operatorname{int}(\mathcal{K})) \neq \emptyset$. Then there exists $\left(\overline{\mathrm{y}}^{*}, \bar{z}^{*}\right) \in \mathrm{B}^{\text {st }} \times \mathcal{K}^{+}$such $\left\langle\bar{z}^{*}, \bar{z}\right\rangle=0,\left(\bar{x}, \bar{y}, \bar{z}, \bar{y}^{*}, \bar{z}^{*}\right)$ is a super maximizer of (MMWD), and the corresponding objective values of (SOP) and (MMWD) are equal.

Proof. Utilizing Theorem 3.10, Lemma 3.17, and Lemma 3.18 in place of Theorem 3.4, Lemma 3.12, and Lemma 3.13, and arguing as in the proof of Theorem 3.15, we can derive the desired conclusion.

\section{Acknowledgment}

This research was supported by Natural Science Foundation of China under Grant No. 11361001. This work was finished when the author was visiting Northern Michigan University. The author would like to extend his sincere gratitude to Qinghong Zhang and Truong Q. Bao in the Department of Mathematics \& Computer Science at Northern Michigan University, USA for their assistance.

\section{References}

[1] T. Q. Bao, B. S. Mordukhovich, Necessary conditions for super minimizers in constrained multiobjective optimization, J. Global Optim., 43 (2009), 533-552. 1

[2] J. M. Borwein, D. Zhuang, Super efficiency in vector optimization, Trans. Amer. Math. Soc., 338 (1993), 105-122. 1

[3] Y. H. Cheng, W. T. Fu, Strong efficiency in a locally convex space, Math. Methods Oper. Res., 50 (1999), 373-384. 1

[4] R. Cristescu, Ordered vector spaces and linear operators, Translated from the Romanian, Abacus Press, Tunbridge, (1976). 1

[5] A. M. Geoffrion, Proper efficiency and the theory of vector maximization, J. Math. Anal. Appl., 22 (1968), 618-630. 1

[6] X.-H. Gong, Connectedness of super efficient solution sets for set-valued maps in Banach spaces, Math. Methods Oper. Res., 44 (1996), 135-145. 1

[7] A. Guerraggio, E. Molho, A. Zaffaroni, On the notion of proper efficiency in vector optimization, J. Optim. Theory Appl., 82 (1994), 1-21. 1

[8] T. X. D. Ha, Optimality conditions for several types of efficient solutions of set-valued optimization problems, Nonlinear analysis and variational problems, Springer Optim. Appl., Springer, New York, 35 (2009), 305-324. 1

[9] Y.-D. Hu, X.-H. Gong, Super efficiency and its scalarization in topological vector space, Acta Math. Appl. Sinica (English Ser.), 16 (2000), 22-26. 1

[10] Z.-F. Li, S.-Y. Wang, Connectedness of super efficient sets in vector optimization of set-valued maps, Set-valued optimization. Math. Methods Oper. Res., 48 (1998), 207-217. 1

[11] L. J. Lin, Optimization of set-valued functions, J. Math. Anal. Appl., 186 (1994), 30-51. 2

[12] A. Mehra, Super efficiency in vector optimization with nearly convexlike set-valued maps, J. Math. Anal. Appl., 276 (2002), 815-832. 1, 3, 3, 3.3, 3.5

[13] W. D. Rong, Y. N. Wu, Characterizations of super efficiency in cone-convexlike vector optimization with set-valued maps, Set-valued optimization, Math. Methods Oper. Res., 48 (1998), 247-258. 1

[14] P. H. Sach, Nearly subconvexlike set-valued maps and vector optimization problems, J. Optim. Theory Appl., 119 (2003), 335-356. 1, 2, 2.7

[15] P. H. Sach, D. S. Kim, G. M. Lee, Strong duality for proper efficiency in vector optimization, J. Optim. Theory Appl., 130 (2006), 139-151. 1

[16] P. H. Sach, L. A. Tuan, Strong duality with proper efficiency in multiobjective optimization involving nonconvex set-valued maps, Numer. Funct. Anal. Optim., 30 (2009), 371-392. 1

[17] T. Weir, B. Mond, Pre-invex functions in multiple objective optimization, J. Math. Anal. Appl., 136 (1988), $29-38.3 .2$

[18] P. Wolfe, A duality theorem for non-linear programming, Quart. Appl. Math., 19 (1961), 239-244. 3.1

[19] L. Y. Xia, J. H. Qiu, Superefficiency in vector optimization with nearly subconvexlike set-valued maps, J. Optim. Theory Appl., 136 (2008), 125-137. 1, 2.1, 2, 2.2, 2.3, 2.4, 2.8, 2.9, 2.12, 3, 3, 3.3, 3.5, 3.3

[20] Y.-H. Xu, S.-Y. Liu, Super efficiency in the nearly cone-subconvexlike vector optimization with set-valued functions, Acta Math. Sci. Ser. B Engl. Ed., 25 (2005), 152-160.

[21] Y.-H. Xu, C.-X. Zhu, On super efficiency in set-valued optimisation in locally convex spaces, Bull. Austral. Math. Soc., 71 (2005), 183-192. 1, 2.10, 2.12

[22] X. M. Yang, D. Li, S. Y. Wang, Near-subconvexlikeness in vector optimization with set-valued functions, J. Optim. Theory Appl., 110 (2001), 413-427. 1, 2, 2.7

[23] X. Y. Zheng, Proper efficiency in locally convex topological vector spaces, J. Optim. Theory Appl., 94 (1997), 469-486. 1

[24] X. Y. Zheng, X. M. Yang, K. L. Teo, Super-efficiency of vector optimization in Banach spaces, J. Math. Anal. Appl., 327 (2007), 453-460. 1 